\title{
Índices espectrais para avaliação da dinâmica sazonal de clorofilas em folhas de Eucalyptus grandis
}

\author{
Laura Camila de Godoy Goergen ${ }^{1 *}$, Julio Cesar Wincher Soares ${ }^{2}$, Elias Fernando Berra ${ }^{3}$, Catize Brandelero ${ }^{2}$, Rudiney Soares Pereira ${ }^{2}$ \\ ${ }^{1}$ Universidade do Estado de Santa Catarina, Av. Luiz de Camões, no 2090, Conta Dinheiro, CEP 88520-000 Lages, SC, Brasil \\ ${ }^{2}$ Universidade Federal de Santa Maria, Avenida Roraima, no 1.000, Campus Universitário, CEP 97105-900 Santa Maria, RS, Brasil \\ ${ }^{3}$ Newcastle University, School of Civil Engineering and Geosciences, CEP NE1 7RU, Newcastle upon Tyne, Reino Unido
}

\author{
*Autor correspondente: \\ lauragoergen@yahoo.com.br \\ Termos para indexação: \\ Sensoriamento remoto \\ Radiometria de campo \\ Teor de clorofila \\ Processo fisiológico \\ Index terms: \\ Remote sensing \\ Field radiometric \\ Chlorophyll content \\ Physiological process
}

Histórico do artigo:

Recebido em 09/01/2015

Aprovado em 25/05/2015

Publicado em 30/06/2015

doi: $10.4336 / 2015$ pfb 3582841

\begin{abstract}
Resumo - O objetivo deste trabalho foi avaliar o desempenho de diferentes índices espectrais - MCARI705, R750/R700, R750/R550 e MTCI - na descrição da dinâmica temporal de clorofilas em folhas isoladas de Eucalyptus grandis, para quatro estações climáticas. $\mathrm{O}$ trabalho foi realizado com base em três parcelas de $600 \mathrm{~m}^{2}$, em um povoamento com 20 meses de idade. Foram coletadas 15 folhas distribuídas em três camadas da copa, totalizando 45 folhas para cada estação. As leituras espectrorradiométricas foram realizadas com o espectrorradiômetro FieldSpec ${ }^{\circledR} 3$, que recobre a faixa espectral de 350 a $2.500 \mathrm{~nm}$. Foi empregada a análise de variância (ANOVA) e o teste de médias Tukey ao nível de 5\% de significância. Para todos os índices espectrais, os valores encontrados na primavera foram diferentes dos encontrados no verão; o mesmo ocorrendo entre outono e inverno.
\end{abstract}

\section{Spectral indices for chlorophyll seasonal dynamics analysis in leaves of Eucalyptus grandis}

\begin{abstract}
The objective of this study was to evaluate the performance of different spectral indices - MCARI705, R750/R700, R750/R550 and MTCI - in the description of chlorophyll seasonal dynamics in detached leaves of Eucalyptus grandis in four seasons. The study was conducted in three plots of $600 \mathrm{~m}^{2}$, in a 20 months old stand. We collected 15 leaves from three layers of canopy, totalizing 45 leaves for each season. It was used a spectroradiometer FieldSpec $\AA 3$, which covers the spectral range from 350 to 2,500 $\mathrm{nm}$. Variance analysis (ANOVA) and Tukey test at 5\% significance level were carried out. For all spectral indices, values observed in spring were different from those from summer; the same occurred between fall and winter.
\end{abstract}

\section{Introdução}

A abordagem tradicional para a determinação de clorofila é com métodos químicos úmidos que envolvem a extração de pigmentos em solventes orgânicos para medidas espectrofotométricas subsequentes. No entanto, tais procedimentos laboratoriais são trabalhosos, demorados, e incluem o método destrutivo de folhas. A alternativa a esses métodos químicos é a reflectância e/ ou espectroscopia de absorção, que são eficientes e não destrutivos (Gitelson et al., 2009).
Durante o processo fotossintético os pigmentos absorvem energia principalmente em uma faixa do espectro eletromagnético que, segundo Ponzoni \& Shimabukuro (2007), encontra-se entre 0,40 a $0,72 \mu \mathrm{m}$, ou seja, no visível (VIS). Desta maneira, a radiância refletida de folhas isoladas ou de dosséis tem sido objeto de estudos para estimativa de pigmentos fotossintéticos como alternativa aos métodos químicos utilizados tradicionalmente, como observado nos trabalhos de Buschman \& Nagel (1993); Gitelson et al. (1996); Blackburn (1998); Formaggio et al. (2002); Dash \& 
Curran (2004) e Gitelson et al. (2009), dentre outros. Esses estudos foram realizados com a utilização de índices espectrais que, de acordo com Formaggio et al. (2002), utilizam bandas espectrais estreitas na forma de soma, razão ou multiplicação, tendo como objetivo minimizar os efeitos adversos como solos, fundo e fatores estruturais, permitindo, assim, a obtenção de uma boa relação na determinação de componentes fotossintéticos da vegetação.

Chapelle \& Kim (1992) e Peñuelas et al. (1995) empregaram índices de reflectância com bandas estreitas na determinação da concentração absoluta e relativa de clorofila a, clorofila b e carotenóides em folhas vegetais. Seus resultados indicam uma boa aplicabilidade dos índices de reflectância para a determinação da concentração absoluta e relativa dos pigmentos e a forte relação com a concentração por unidade de área de pigmentos individuais em dosséis.

Nos últimos anos, o maior agente de transformação da paisagem no Estado do Rio Grande do Sul são os cultivos florestais, principalmente os cultivos do gênero Eucalyptus. A intensificação da silvicultura demanda a concepção de soluções técnicas de monitoramento dos povoamentos que tenham rápida aplicabilidade e baixo custo, e que sejam capazes de apoiar os procedimentos de condução dos povoamentos. Desta maneira, o sensoriamento remoto hiperespectral (HRS), em nível de campo, apresenta um grande potencial para ser utilizado na silvicultura. Através desta técnica, é possível efetuar predições sobre a produção florestal e mensurar o vigor vegetativo das espécies.

Diante desse contexto, o presente trabalho foi norteado pela hipótese de que os índices espectrais poderão ajudar na determinação do estado fisiológico das plantas e determinar o início da estação de máxima produção fotossintética de Eucalyptus grandis (Hill ex Maiden). Assim, foi delineado como objetivo avaliar o desempenho de diferentes índices espectrais na descrição da dinâmica temporal de clorofilas em folhas isoladas da espécie, para as quatro estações climáticas.

\section{Material e métodos}

\section{Monitoramento florestal}

O trabalho foi realizado na Estação Experimental de Silvicultura de Santa Maria, que está inserida na área de tensão ecológica com atividades agrárias em contato com a Mata Atlântica e o Pampa (IBGE, 2004), localizada no Estado do Rio Grande do Sul, Brasil, entre as coordenadas UTM (Universal Transversa de Mercator) 217.000 e $220.000 \mathrm{mE}, 6.713 .000$ e 6.717 .000 $\mathrm{mN}$ (Fuso $22 \mathrm{~J}$ ).

Aárea encontra-se sob o domínio do clima subtropical úmido do tipo $\mathrm{Cfa}$, com chuvas bem distribuídas ao longo do ano, com precipitação média anual de $1.546 \mathrm{~mm}$ e temperatura média anual de $19^{\circ} \mathrm{C}$ (Moreno, 1961).

Foi selecionado um talhão em um povoamento de Eucalyptus grandis (Hill ex Maiden) com 20 meses de idade, implantado sob relevo suave ondulado (Figura 1), em solo classificado como Argissolo Vermelho distrófico úmbrico (Santos et al., 2006) ou Paleudult (Soil Survey Staff, 1999).

Os procedimentos amostrais consistiram na instalação de três parcelas de $600 \mathrm{~m}^{2}$, sendo selecionada uma árvore de posição central no interior de cada uma delas, onde foram efetuadas coletas de 15 folhas distribuídas ao longo da copa, totalizando 45 folhas para cada estação.

\section{Espectrorradiometria e análises estatísticas}

As leituras espectrorradiométricas foram realizadas no Laboratório de Sensoriamento Remoto da Universidade Federal de Santa Maria, com o espectrorradiômetro FieldSpec ${ }^{\circledR} 3$, acoplado à unidade RTS-3ZC3 (esfera integradora), revestida internamente pelo material Spectralon que se assemelha a uma superfície lambertiana, perfeitamente difusa e que reflete aproximadamente $100 \%$ em todo o espectro solar. Essas características da esfera integradora permite que se obtenha o fator de reflectância, pela razão entre a radiância refletida pelo objeto lido e a radiância refletida pela placa Spectralon.

Os procedimentos de leitura tiveram início uma hora após o material vegetativo ter sido coletado. $\mathrm{O}$ espectrorradiômetro realiza medições de irradiação, radiância solar e fator de reflectância (FR), sendo um aparelho para uso em campo e laboratório. Possui uma resolução espectral de $10 \mathrm{~nm}$ e capacidade de coletar até 10 espectros por segundo.

Cada folha foi posicionada no orifício da esfera (espectrorradiômetro), com face ventral voltada para o interior da esfera, sendo realizadas 10 leituras por segundo. Os espectros gerados foram transferidos para o microcomputador e gravados em arquivo para o pósprocessamento.

Os dados resultantes desse procedimento constituem um conjunto de arquivos contendo comprimento de onda 
e o FR correspondente na amplitude de $350 \mathrm{nma} 2.500 \mathrm{~nm}$. Posteriormente, foram isolados os dados relativos ao FR entre $550 \mathrm{~nm}$ a $750 \mathrm{~nm}$. Essas rotinas foram realizadas utilizando os aplicativos $\mathrm{RS}^{3}{ }^{\circledR} 160521$ (software de interface com o usuário para aquisição e armazenamento de dados espectrais), ASD ViewSpecPro ${ }^{\circledR}$ Versão 4.05 e planilha eletrônica (Figura 1).
Posteriormente, os dados de FR foram submetidos à análise exploratória e ao teste de normalidade no software R 2.15.1. Os dados apresentaram distribuição normal, desta maneira, foi empregada a análise de variância (ANOVA). Quando foram observadas diferenças significativas, as médias foram comparadas pelo teste de Tukey ao nível de 5\%.

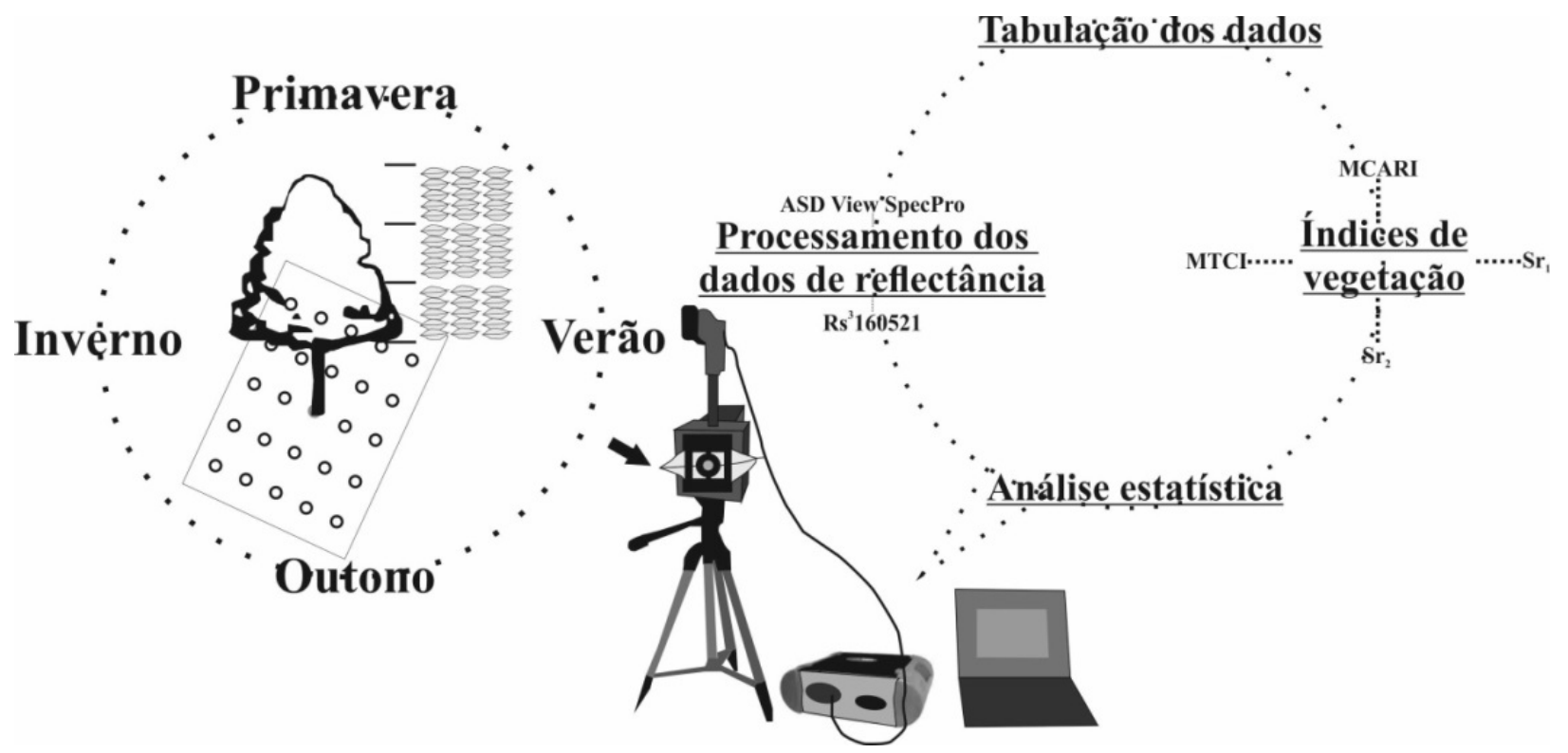

Figura 1. Esquema metodológico das etapas e processos envolvidos no estudo.

\section{Índices espectrais utilizados}

A seguir são descritos os índices espectrais selecionados que, segundo seus autores, apresentaram alta correlação com o teor de clorofila para as mais diversas culturas.

\section{Índice MCARI ${ }_{705}$}

O Modified Chlorophyll Absorption Ratio index (MCARI) proposto por $\mathrm{Wu}$ et al. (2008), apresenta forte relação com aos comprimentos de onda 705 e $750 \mathrm{~nm}$. Usando bandas hiperespectrais estreitas, os autores encontraram uma relação direta entre o índice proposto e o conteúdo de clorofila em folhas de trigo, com coeficiente de determinação $\left(\mathrm{R}^{2}\right)$ de 0,70 . Este índice é descrito na Equação 1, onde: $\mathrm{R}_{\mathrm{x}}$ é a reflectância no comprimento de onda $\mathrm{x}(\mathrm{nm})$ :

$$
\operatorname{MCARI}_{350}=\left[\left(\mathrm{R}_{350}-\mathrm{R}_{730}\right)-0.2\left(\mathrm{R}_{350}-\mathrm{R}_{350}\right)\right]\left(\frac{\mathrm{R}_{730}}{\mathrm{R}_{305}}\right)
$$

\section{Índice Razões de Reflectância $\mathbf{R}_{750} / \mathbf{R}_{700}$ e $\mathbf{R}_{750} / \mathbf{R}_{550}$}

Gitelson et al. (1996) buscaram um comprimento de onda em que a clorofila fosse o único fator que influenciasse na reflectância. Assim, os autores observaram que a reflectância próximo a $700 \mathrm{~nm}$ e no intervalo de 530 a $630 \mathrm{~nm}$ foram sensíveis ao conteúdo de clorofila. Os autores também observaram que o intervalo de comprimento de onda entre 750 e $900 \mathrm{~nm}$ pode ser considerado como insensível ao teor de clorofila. Tendo em vista que $\mathrm{R}_{700}$ e $\mathrm{R}_{550}$ são diretamente proporcionais ao teor de clorofila, e que $\mathrm{R}_{750}$ praticamente não depende do teor de clorofila, foram desenvolvidos os índices de razão simples (SR - Simple Ratio), descritos nas equações 2 e 3 .

$$
\begin{gathered}
\mathrm{SR}_{1}=\frac{\mathrm{R} 750}{\mathrm{R} 700} \\
\mathrm{SR}_{2}=\frac{\mathrm{R} 750}{\mathrm{R} 550}
\end{gathered}
$$




\section{Índice MTCI}

Dash \& Curran (2004) desenvolveram o índice MERIS Terrestrial Chlorophyll Index (MTCI), utilizando dados de reflectância do vermelho próximo (espectrorradiômetro de média resolução) para a predição do teor de clorofila em dosséis florestais. Os autores observaram que com o aumento do teor de clorofila a diferença na reflectância entre 750 e $710 \mathrm{~nm}$ diminuiu gradualmente, aumentando entre os comprimentos de onda 710 e $680 \mathrm{~nm}$. A partir dessas análises foi gerado o índice MTCI (Equação 4).

$$
M T C=\left(\frac{R 750 \cdot R 710}{R 710 \cdot R 680}\right)
$$

\section{Resultados e discussão}

Os valores médios dos índices espectrais para as quatro estações climáticas estão apresentados na Fig. 2. Para todos os índices espectrais, os valores encontrados na primavera foram diferentes dos encontrados no verão; o mesmo ocorrendo entre outono e inverno. Já a transição verão-outono, em três dos quatro índices, não acarretou diferenças entre os índices.

Ao se observar a variação das estações climáticas em sua ordem cronológica percebe-se uma tendência sazonal nos gráficos. Para o índice MCARI ocorrem valores mais baixos no inverno-primavera, aumentando no verão-outono e com SR1, SR2 e MTCI os menores valores ocorrem no verão-outono, aumentando no inverno-primavera. Lembrando que estes índices foram concebidos para serem sensíveis ao conteúdo de clorofila de maneira direta com MCARI e inversa com SR1, SR2 e MTCI (Gitelson et al., 1996; Dash \& Curran, 2004; Wu et al., 2008). Assim, pode-se inferir que as folhas analisadas apresentam maior conteúdo de clorofila no período verão-outono e menor conteúdo no período inverno-primavera.

De acordo com Wu et al. (2008), as clorofilas têm fortes picos de reflectância nas regiões de comprimento de onda do verde. Porém, segundo os autores, a alta reflectância encontrada no espectro do azul não pode ser aproveitada para a predição do teor de clorofila, uma vez que há a sobreposição com a absortância $(\alpha)$ dos carotenóides. A absorção máxima na região do vermelho ocorre entre 660 e $680 \mathrm{~nm}$. No entanto, é desconhecido se a reflectância nestes comprimentos de onda pode ser usada para predizer o teor de clorofila, pois a absorção em 660 e $680 \mathrm{~nm}$ tende a saturar a baixo teor de clorofila, reduzindo assim a sensibilidade dos índices espectrais baseados nestes comprimentos de onda (Sims \& Gamon, 2002).

Os dados de reflectância espectral foram submetidos à análise de variância (ANOVA), demonstrando que houve diferenças nos teores de clorofilas das folhas de Eucalyptus grandis em função das estações climáticas (Tabela 1).

Tabela 1. ANOVA's aplicadas aos índices espectrais para folhas de Eucalyptus grandis com 20 meses de idade.

\begin{tabular}{clcccc}
\hline Índice & & $\mathrm{gl}$ & $\mathrm{SQ}$ & $\mathrm{QM}$ & $\mathrm{F}$ \\
\hline MCARI & Estações & 3 & 0,08755 & 0,02918 & $21,45^{* *}$ \\
& Resíduos & 176 & 0,23940 & 0,00136 & \\
SR2 & Estações & 3 & 28,87 & 9,623 & $35,22 * *$ \\
& Resíduos & 176 & 48,09 & 0,273 & \\
SR1 & Estações & 3 & 14,33 & 4,775 & $15,05^{* *}$ \\
& Resíduos & 176 & 55,83 & 0,317 & \\
MTCI & Estações & 3 & 18,36 & 6,119 & $30,92 * *$ \\
& Resíduos & 176 & 34,83 & 0,198 & \\
\hline
\end{tabular}

** Significativo ao nível de confiança de $99 \% ; \mathrm{gl}=$ graus de liberdade; $\mathrm{SQ}=$ soma de quadrados; $\mathrm{QM}=$ quadrado médio; $\mathrm{F}=$ valor de F calculado. 

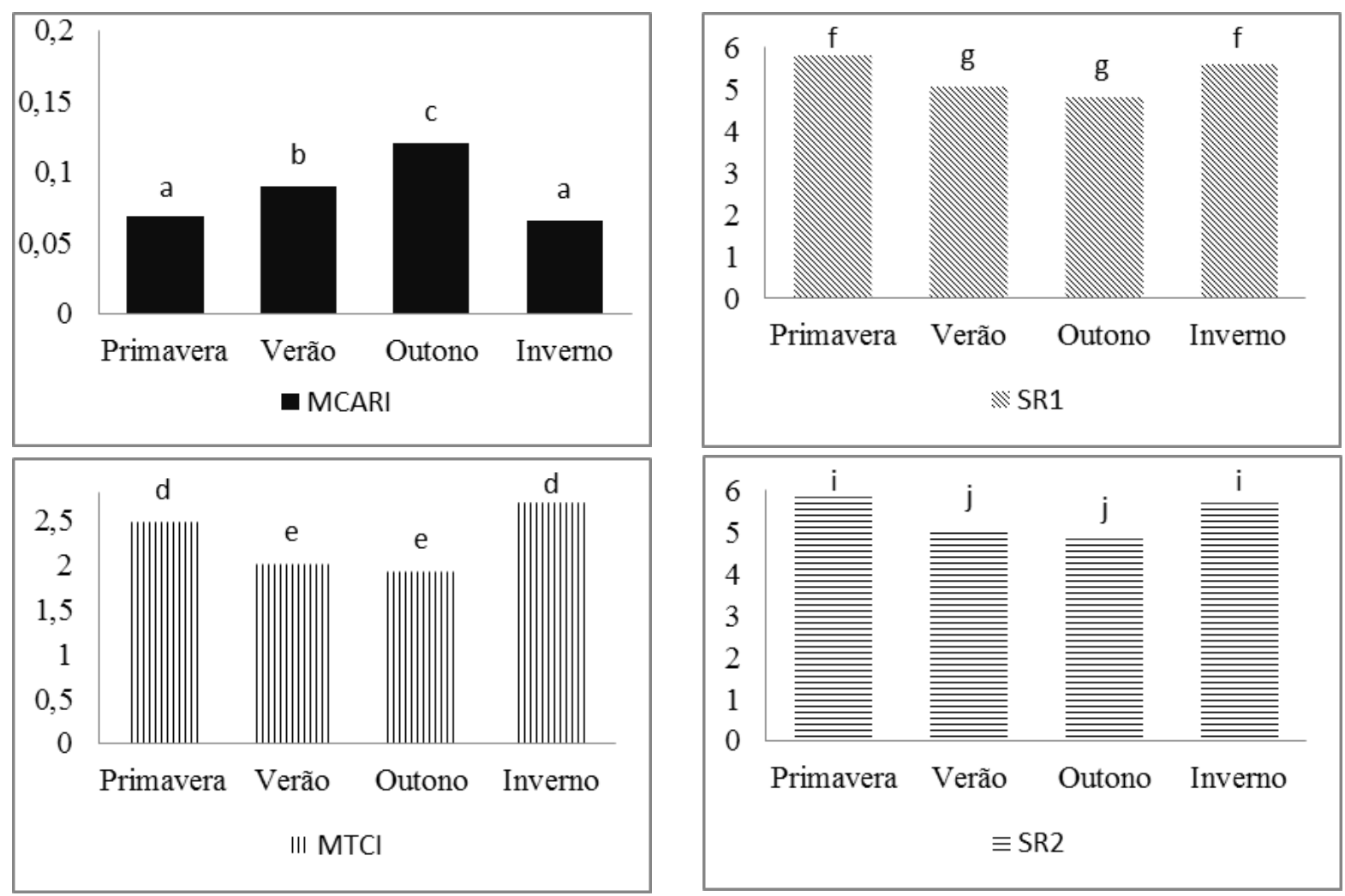

Figura 2. Valores médios (adimensionais) dos índices espectrais em folhas de Eucalyptus grandis para as quatro estações climáticas. Médias seguidas de letras iguais entre as estações não diferem significativamente entre si pelo teste de Tukey a 5\% de probabilidade.

Apesar de não haver diferenças nos valores dos índices SR1, SR2 e MTCI entre verão-outono, ocorreu valor médio menor no outono. MCARI apresentou valor maior no outono, sendo que este difere do verão. Estes eventos sugerem que o maior teor de clorofila ocorreu no outono. Gitelson \& Merzlyak (1997) encontraram para os índices SR1 e SR2 forte correlação entre o conteúdo de clorofila predito e o determinado por método químico, para as folhas de diversas espécies arbóreas. Formaggio et al. (2002), encontraram $R^{2}$ maior que 0,77 com a razão R750/R550, para estudos de clorofila em dosséis de soja.

Freiberger et al. (2010) realizaram estudos de concentração de clorofila em folhas de Anadenanthera colubrina (Angico branco) e observaram que no final do inverno e da primavera existiam concentrações menores de clorofila a e b, havendo aumento destas concentrações no final do verão. Os mesmos autores encontraram teor total de clorofila constante para Tabebuia avellanedae (ipê-roxo), independentemente da estação climática. Este comportamento encontra respaldo em estudos realizados por Almeida et al. (2004), os quais afirmam que, para algumas espécies, pode ocorrer um maior acúmulo de clorofilas sob baixa radiação solar devido ao efeito compensatório a menor quantidade de radiação disponível.

Demarez et al. (1999) observaram que os teores de clorofila aumentaram a partir de meados do inverno em direção à primavera e no início de verão em três espécies arbóreas europeias. Berveiller \& Damesin (2008) também constataram valores máximos de clorofila e taxa fotossintética no início do período vegetativo, ou seja, na primavera, em ramos novos de Fagus sylvatica (faia-européia).

Segundo Gabrielsen (1948), a proporção de luz incidente que é refletida é maior quando a concentração de pigmentos na folha é baixa, porque a luz refletida de uma camada mais interna terá menor chance de ser recapturada no seu caminho de volta à superfície. Ainda para o mesmo autor, quanto menor a concentração de clorofila na folha, maior será o aumento relativo da absorção de luz devido a um aumento nesta concentração inicial. Esta relação pode ser observada também para a clorofila extraída, embora as propriedades óticas das folhas não sejam as mesmas que a da clorofila em 
solução. Ciganda et al. (2008) observaram teor maior de clorofila na parte inferior do dossel de milho (Zea mays L.). Essa ocorrência, segundo os autores, está relacionada a uma redução na capacidade de transporte de elétrons por unidade de clorofila presente, devido à baixa incidência de radiação solar.

Com o emprego dos índices MCARI705 e SR705, Mielke et al. (2012) obtiveram boas predições para o conteúdo de clorofila nas folhas de Eugenia uniflora, determinando valores do $\mathrm{r}^{2}$ de 0,94 e 0,93 , respectivamente.

O aumento da concentração de clorofila está relacionado ao aumento da atividade fotossintética. Inoue \& Martins (2006) observaram que a variação da taxa fotossintética da grevílea seguiu a alteração da temperatura. Os autores registraram o incremento sazonal: inverno $>$ primavera $>$ verão, retornando aos níveis iniciais do outono do período anterior. Tal comportamento também foi verificado em diferentes espécies lenhosas (Machado et al., 2002; Alves, 2004), em que a fotossíntese foi influenciada pelas estações do ano. Considerando que a espécie estudada é heliófita, e que houve diferença na resposta espectral entre a estação com maior intensidade luminosa (verão), pode-se inferir que esta responde fisiologicamente a essa característica, apontando assim a ocorrência de diferentes concentrações de pigmentos nas diferentes épocas do ano.

\section{Conclusões}

Os índices espectrais avaliados apresentaram variações para as estações climáticas monitoradas, o que sugere a correlação existente entre eles e o teor de clorofila nas folhas. Isso torna possível o monitoramento da cultura através de técnicas de sensoriamento remoto.

Os resultados encontrados para os índices de reflectância apontam que a estação de máxima produção fotossintética do Eucalyptus grandis ocorre no período verão-outono, onde se encontra maior conteúdo de clorofila nas folhas.

\section{Referências}

ALMEIDA, L. P.; ALVARENGA, A. A.; CASTRO, E. M.; ZANELA, S. M.; VIEIRA, C. V. Crescimento inicial de plantas de Cryptocaria aschersoniana Mez. submetidas a níveis de radiação solar. Ciência Rural, Santa Maria, RS, n. 34, p. 83-88, 2004.

Pesq. flor. bras., Colombo, v. 35, n. 82, p. 55-61, abr../jun. 2015
ALVES, A. O. Estudo da fotossíntese de espécies dominantes em floresta de transição no sudoeste da Amazônia. 2004.77 f. Dissertação (Mestrado em Física e Meio Ambiente) - Instituto de Ciências Exatas e da Terra, Universidade Federal de Mato Grosso, Cuiabá.

BERVEILLER, D.; DAMESIN, C. Carbon assimilation by tree stems: potential involvement of phosphoenolpiruvate carboxilase. Trees: Structure and Function. Vancouver, v. 22, n. 2, p. 149-157, 2008.

BLACKBURN, G. A. Quantifying chlorophylls and carotenoids at leaf and canopy scales: an evaluation of some hyper-spectral approaches. Remote Sensing of Environment, Florida, n. 66, p. 273-285, 1998.

BUSCHMAN, C.; NAGEL, E. In vivo spectroscopy and internal optics of leaves as a basis for remote sensing of vegetation. International Journal of Remote Sensing, Virginia, n. 14, p. 711-722, 1993.

CHAPELLE, E. W.; KIM, M. S. Ratio analysis of reflectance spectra (RARS): an algorithm for the remote estimation of the concentrations of chlorophyll a, chlorophyll $\mathrm{b}$ and the carotenoids in soybean leaves. Remote Sensing of Environment, Florida, n. 39, p. 239-247, 1992

CIGANDA, V.; GITELSON, A.; SCHEPERS, J. Vertical profile and temporal variation of chlorophyll in maize canopy: quantitative "crop vigor" indicator by means of reflectancebased techniques. Agronomy Journal, Lincoln, n. 100, p. 1409-1417, 2008.

DASH, J.; CURRAN, P. J. The MERIS terrestrial chlorophyll index. International Journal of Remote Sensing, Virginia, n. 25, p. 5403-5413, 2004.

DEMAREZ, V.; GASTELLU-ETCHEGORRY, J. P.; MOUGIN, E.; MARTY, G.; PROISY, C. Seasonal variation of leaf chlorophyll content of a temperate forest. Inversion of the PROPECT model. International Journal of Remote Sensing, Virginia, n. 20, p. 879894, 1999.

FORMAGGIO, A. R.; FERRI, C. P.; SCHIAVINATO, M. A. Comparação entre índices espectrais de vegetação para determinação de clorofila em dosséis de soja [Glycine max (L.), Merri]. In: SIMPOSIO LATINOAMERICANO DE PERCEPCION REMOTA Y SISTEMAS DE INFORMACION ESPACIAL, 10., 2002. Cochabamba, Bolivia. Anais... Cochabamba: Universidade Maior de San Simon: Clas, 2002. CD-ROM.

FREIBERGER, M. B.; CASTOLDI, G.; MARINI, D.; LANG, A.; HERZOG, N. F. M.; MALAVASI, U. C. Variação sazonal de clorofilas em folhas de Tabebuia avellanedae e Anadenanthera colubrina. Semina, Londrina, n. 31, p. 1343-1348, 2010.

GABRIELSEN, E. K. Effects of different chlorophyll concentrations on photosynthesis in foliage leaves. Physiologia Plantarum, Lund, n. 1, p. 5-37, 1948.

GITELSON, A.; KAUFMAN, Y. J.; MERZLYAK, M. N. Use of a green channel in remote sensing of global vegetation from EOSMODIS. Remote Sensing of Environment, Florida, n. 58, p. 289-298, 1996.

GITELSON, A.; CHIVKUNOVA, O. B.; MERZLYAK, M. N. Nondestructive estimation of anthocyanins and chlorophylls in anthocyanic leaves. American Journal of Botany, St. Louis, n. 96, p. 1861-1868, 2009. 
GITELSON, A.; MERZLYAK, M. N. Remote estimation os chlorophyll content in higher plant leaves. International Journal of Remote Sensing, Virginia, n. 18, p. 2691-2697, 1997.

IBGE. Mapa de vegetação do Brasil. Escala 1:5.000.000. 2004.

INOUE, M. T.; MARTINS, E. G. Variação sazonal da fotossíntese e clorofila em progênies de Grevillea robusta Cunn. Revista Ciências Exatas e Naturais, Guarapuava, n. 8, p. 113-124, 2006.

MACHADO, E. C.; MEDINA, C. L.; GOMES, M. M. A.; HABERMANN, G. Variação sazonal da fotossíntese, condutância estomática e potencial de água na folha de laranjeira 'Valência'. Scientia Agricola, Piracicaba, n. 59, p. 53-58, 2002.

MIELKE, M. S.; SCHAFFER, B.; SCHILLING, A. C. Evaluation of reflectance spectroscopy indices for estimation of chlorophyll content in leaves of a tropical tree species. Photosynthetica, Praga, n. 50, p. 343-352, 2012.

MORENO, J. A. Clima do Rio Grande do Sul. Porto Alegre: Secretaria da Agricultura, Diretoria de Terras e Colonização. Seção de Geografia, 1961. 42 p.

PEÑUELAS, J.; BARET, F.; FILELLA, I. Semi-empirical indices to assess carotenoids/chlorophyll a ratio from leaf spectral reflectance. Photosynthetica, Praga, n. 31, p. 221-230, 1995.
PONZONI, F. J.; SHIMABUKURO, Y. E. Sensoriamento remoto no estudo da vegetação. São José dos Campos: Parêntese, 2007.

SANTOS, H. G. dos; JACOMINE, P. K. T.; ANJOS, L. H. C. dos; OLIVEIRA, V. A. de; OLIVEIRA, J. B. de; COELHO, M. R.; LUMBRERAS, J. F.; CUNHA, T. J. F. (Ed.). Sistema brasileiro de classificação de solos. 2. ed. Rio de Janeiro: Embrapa Solos, 2006. 306 p.

SIMS, D. A.; GAMON, J. A. Relationships between leaf pigment content and spectral reflectance across a wide range of species, leaf structures and developmental stages. Remote Sensing of Environment, Florida, n. 81, p. 337-354, 2002.

SOIL SURVEY STAFF (Washington, D.C.). Soil taxonomy: a basic system of soil classification for making and interpreting soil surveys. 2nd ed. Washington, D.C.: USDA, Natural Resources Conservation Service, 1999. (USDA. Agriculture Handbook, 436).

WU, C.; NIU, Z.; TANG, Q.; HUANG, W. Estimating chlorophyll content from hyperspectral vegetation indices: modeling and validation. Agricultural and Forest Meteorology, New Haven, n. 148, p. 1230-1241, 2008. 
